\title{
Estimação de Índices de Aprovação e Reprovação Escolar do Ensino Médio
}

\author{
Ricardo B. das Neves Junior ${ }^{1}$, Rafaella L. S. do Nascimento ${ }^{2}$, Roberta A. A. Fagundes ${ }^{1}$, \\ Paulo S. G. de Mattos Neto ${ }^{2}$ \\ ${ }^{1}$ Escola Politécnica de Pernambuco, Universidade de Pernambuco (UPE) \\ R. Benfica, 455 - Madalena, Recife PE, 50720-001 \\ ${ }^{2}$ Centro de Informática, Universidade Federal de Pernambuco (UFPE) \\ Av. Jorn. Aníbal Fernandes, s/n - Cidade Universitária, Recife - \\ rbnj@ecomp.poli.br, rlsn@cin.ufpe.br, roberta.fagundes@upe.br, psgmn@cin.ufpe.br
}

\begin{abstract}
Educational data mining seeks to study and contribute results that explain variables and find possible solutions to problems in the area of education. Considering this motivation, this article describes a study based on educational data provided by INEP and the construction of prediction models using nonparametric quantile regression with and without parameter optimizer. We present a descriptive study of the explanatory variables of the model, which is used to predict school approval and disapproval. The results obtained show that the quantile regression with optimization received a smaller prediction error. The study shows the relevance in the application of nonparametric regression techniques.
\end{abstract}

Resumo. A mineração de dados educacionais busca estudar e contribuir com resultados que expliquem variáveis e encontrem possíveis soluções para problemas na área da educação. Tendo em vista essa motivação, este artigo descreve um estudo com base em dados educacionais fornecidos pelo INEP e a construção de modelos de predição utilizando a regressão quantílica não paramétrica com e sem otimizador de parâmetros. Apresenta-se um estudo descritivo das variáveis explicativas do modelo o qual é utilizado para predizer a aprovação e a reprovação escolar. Os resultados obtidos mostram que a regressão quantílica com otimização obteve menor erro de predição. O estudo mostram a relevância na aplicação de técnicas não paramétricas de regressão.

\section{Introdução}

O estudo de variáveis educacionais é uma corrente e objeto de várias pesquisas, com o objetivo de compreender o contexto do fenômeno nos mais diferentes aspectos do ensino como ambientes educacionais, modalidades (à distância e presencial) e estágios da educação. Um dos fatores que facilitam o desenvolvimento destes trabalhos é a disponibilidade de dados relacionados à educação, aumentando a aplicabilidade da Mineração de Dados Educacionais (MDE) [Romero and Ventura 2010].

A MDE é uma abordagem que objetiva a descoberta de informações que ajudem na proposta educacional, no melhoramento das condições de infraestrutura escolar, no processo ensino, na previsão de desempenho dos alunos, além de outros fatores que 
VIII Congresso Brasileiro de Informática na Educação (CBIE 2019)

Anais do XXX Simpósio Brasileiro de Informática na Educação (SBIE 2019)

influenciam a aprendizagem e que representam a qualidade do ensino, como os indicadores educacionais. Os indicadores educacionais atribuem valor estatístico à qualidade do ensino, atendo-se não somente ao desempenho dos alunos, mas também ao contexto econômico e social em que as escolas estão inseridas [INEP 2019].

De forma geral, a MDE explora técnicas estatísticas, de aprendizado de máquina e de mineração de dados (MD) sobre os diferentes tipos de dados educacionais. Existem várias linhas de pesquisa na área de educação e muitas delas derivadas da área de MD, como tarefas preditivas, de agrupamento ou de associação [Baker et al. 2011]. Entre as preditivas, podem ser utilizadas técnicas de regressão, que têm como um dos objetivos predizer o valor da variável resposta a partir da informação proveniente de uma variável ou de um conjunto de variáveis explicativas [Montgomery et al. 2012].

Estas técnicas são abordadas neste trabalho na estimação dos indicadores educacionais da reprovação e aprovação escolar de alunos do ensino médio no âmbito do estado de Pernambuco. As bases de dados utilizadas na pesquisa são fornecidas abertamente pelo Instituto Nacional de Estudos e Pesquisas Educacionais Anísio Teixeira - INEP e são referentes ao ano de 2016. Dentre as possíveis técnicas de regressão, investiga-se a aplicação da abordagem não paramétrica a partir da regressão quantílica.

A regressão quantílica difere dos demais tipos de regressão, pois permite o uso de vários quantis para obter uma visão mais completa da relação entre as variáveis estudadas [Koenker and Bassett Jr 1978]. Na regressão quantílica não paramétrica (RQNP), um kernel pode ser aplicado ajustando a largura de banda (parâmetro que controla o grau de suavidade da função estimada). Investiga-se, portanto, o ganho que essas técnicas podem oferecer para a área educacional em direção a uma melhor resposta de predição.

Este artigo está dividido da seguinte forma: o item 2 apresenta trabalhos relacionados ao tema deste artigo; em 3 apresenta a base de dados utilizada bem como o processamento realizado; em 4, os modelos propostos; 5 mostra a avaliação experimental e os resultados obtidos; e a seção 6 compõe as conclusões, desenvolvidas após a análise dos resultados obtidos ao final dos experimentos.

\section{Trabalhos Relacionados}

No trabalho de [Laisa and Nunes 2015] o objetivo foi analisar uma base dados de alunos do ensino médio dos anos de 2011 a 2014, por série, a partir da utilização da técnica de classificação usando o Algoritmo J48. As bases obtidas foram de uma escola particular. Busca entender as variáveis relacionadas aos alunos aprovados e reprovados. Os modelos gerados utilizaram-se dos seguintes atributos de cada aluno: nível ( $1^{\mathrm{a}}, 2^{\mathrm{a}}$ ou $3^{\mathrm{a}}$ série), cidade de origem (Local ou Vizinha), bolsista (sim ou não), desistentes (sim ou não), sexo do aluno, idade e ano (2011 a 2014).

O trabalho de [do Nascimento et al. 2018a] utiliza bases de dados educacionais fornecidas pelo INEP e aplica técnicas de mineração de dados com a finalidade de melhor explicar indicadores educacionais como a evasão e reprovação escolar no ensino fundamental. Realiza análise correlacional entre variáveis e aplica modelos de regressões linear e robusta para predição das variáveis resposta. Estas bases do INEP são utilizadas em [Colpani 2018], com dados do ano de 2017. Aplicou-se regressão linear a fim de analisar as variáveis que se relacionam com a evasão escolar. Como resultados, foi possível verificar que a taxa de distorção idade-série apresentou a maior correlação positiva. 
VIII Congresso Brasileiro de Informática na Educação (CBIE 2019)

Anais do XXX Simpósio Brasileiro de Informática na Educação (SBIE 2019)

A pesquisa desenvolvida por [do Nascimento et al. 2018b] utiliza duas técnicas não paramétricas de regressão, Regressão Quantílica e SVR, para prever a evasão no estado de Pernambuco, para alunos do ensino fundamental. O desenvolvimento do trabalho seguiu as fases do CRISP-DM (Cross-Industry Standard for Data Mining) e utilizou os dados do Censo Escolar de 2015. Algumas variáveis explicativas utilizadas são: número de computadores, de salas, de funcionários e localização da escola.

No trabalho de [Calixto et al. 2017] objetivo foi identificar as variáveis relacionadas à evasão escolar, utilizando os dados do Censo Escolar dos anos de 2014, 2015 e 2016 dos estados de Ceará e Sergipe. Aplicou-se técnicas de Indução de Regras e Regressão Logística. Como resultados obtidos, a idade, etapa de ensino, modalidade de ensino, existência de laboratórios e localização da escola se destacaram como variáveis influentes na evasão escolar no cenário de estudo.

Para realizar a estimação dos índices de aprovação e reprovação em escolas, este trabalho utiliza uma abordagem de RQNP utilizada por [Li et al. 2013]. O modelo proposto pelos autores apresenta um método automático para estimação do parâmetro Largura de Banda presente na RQNP baseado em validação cruzada. O método está disponível no R ( $R$ Core Team) através do pacote "np"(função "npcdistbw") versão 0.4014 ou superior [Hayfield and Racine 2008]. Como segunda alternativa de estimação do parâmetro Largura de Banda presente na RQNP, este trabalho utiliza os Algoritmos Genéticos (AG), fundamentados por [Holland 1992], os AGs são algoritmos inteligentes de otimização da família da computação evolucionária e são inspirados na teoria das origens das espécies proposta por Darwin (1909) [Darwin 1909].

Tendo em vista estes trabalhos relacionados, esta pesquisa traz como diferencial a aplicação de técnicas de regressão não paramétricas, enquanto a maioria dos estudos com indicadores educacionais consistem em aplicar modelos de regressão paramétricos ou de classificação. Assim, utilizando a técnica de RQNP, serão construídas as análises preditivas da aprovação e reprovação escolar. As técnicas não paramétricas trazem uma modelagem mais flexível e busca uma resposta que melhor represente os dados avaliados.

\section{Bases de Dados}

As bases de dados utilizadas neste estudo são disponibilizadas abertamente pelo INEP [INEP 2019]. Os dados são referentes a indicadores educacionais do ano de 2016. Os indicadores educacionais considerados são:

- Taxa de eficiência escolar (TR/TA): Taxa de Reprovação/Aprovação da escola.

- Adequação da formação de professores (AFD): Porcentagem de professores por grupo da adequação da formação à disciplina que ensinam (cinco grupos, AFD11 a AFD5).

- Alunos por turma (ATU): O número médio de alunos por turma.

- Complexidade da gestão (ICG): Nível de complexidade de gestão escolar. O indicador classifica as escolas em níveis de 1 a 6 de acordo com sua complexidade de gestão, níveis elevados indicam maior complexidade.

- Distorção idade-série (TDI): Taxa de distorção idade-série dos alunos por escola.

- Professor com ensino superior (DSU): O percentual de professores com ensino superior na escola. 
VIII Congresso Brasileiro de Informática na Educação (CBIE 2019)

Anais do XXX Simpósio Brasileiro de Informática na Educação (SBIE 2019)

- Esforço do professor (IED): O percentual de professores que trabalham no ensino fundamental e médio pelo nível de esforço exigido para o exercício da profissão (seis níveis, IED1 a IED6).

- Média de horas de aula (HAU): O número médio de horas/aulas diário da escola.

- A regularidade do professor (IRD): A média do indicador de regularidade do professor.

Todas essas variáveis foram integradas em um base de dados únicas, levando em consideração o identificador único de cada escola. Nessas bases, os indicadores de eficiência escolar como reprovação e aprovação são as variáveis resposta (y), e os demais são as variáveis explicativas $(x)$.

\subsection{Pré-processamento e Tratamento dos Dados}

Como a base inclui informações sobre todos os estados do Brasil, foi realizada a extração de dados apenas para o estado de Pernambuco, o qual é o alvo desta pesquisa. A base também informa sobre várias etapas do ensino, como fundamental e médio. Considerouse a divisão em duas bases distintas, uma para o ensino médio e outra para o ensino fundamental. No entanto, nesta aplicação utiliza-se os dados relacionados ao ensino médio. Isto significa que busca-se estimar os valores de de Reprovação e Aprovação escolar no ensino médio das escolas do estado de Pernambuco a partir dos indicadores educacionais.

Após isso, foi possível observar poucos valores ausentes para algumas variáveis explicativas, e para resolver esse problema, foi realizada a inserção de valores utilizando a mediana dos valores das colunas. Isto foi realizado uma vez que o objetivo foi ter o mínimo de perda de instâncias. Diferente disso, a variável HAU, a qual se refere a média de horas-aula na escola, continha um grande número de valores faltantes. Optou-se pela exclusão da HAU da base de dados pois utilizar estratégia de resolução de missing value alteraria a variabilidade deste indicador. Nessa fase, também foi aplicada a padronização dos dados, a qual consiste em ajustar a escala dos valores dos atributos para que os valores fiquem em pequenos intervalos, tais como entre 0 a 1 . Portanto, no final deste período a base é composta por 16 variáveis explicativas, em que a base de reprovação possui 1.012 instâncias, enquanto a base de aprovação possui 1.135 instâncias.

\subsection{Seleção de Variáveis}

Para selecionar quais variáveis explicativas seriam utilizadas na execução das previsões, foi escolhido o algoritmo Random Forest (RF) proposto por Breiman em 2001 [Breiman 2001]. Embora o objetivo inicial do algoritmo, fosse desempenhar tarefas de classificação, a literatura indica que o RF é capaz de executar atividades de seleção de variáveis, gerando um rank das variáveis de acordo com a importância em relação à variável dependente, ou seja, as variáveis mais importantes deverão ser utilizadas no modelos de previsão [Coutinho 2015]. As 5 variáveis mais importantes selecionadas pelo RF foram utilizadas no modelo de previsão, estas variáveis são: TDI, AFD1, ATU, AFD3, AFD5 (conjunto de dados Aprovação do Ensino Médio) e TDI, AFD3, ATU, AFD1, IRD (conjunto de dados Reprovação do Ensino Médio).

Como pode ser visto, nos dois cenários há a seleção das mesmas variáveis, em maioria. A distorção idade-série é um dos grandes problemas na área da educação, e possui como uma das principais causas a reprovação e o abandono escolar, fazendo com que 
VIII Congresso Brasileiro de Informática na Educação (CBIE 2019)

Anais do XXX Simpósio Brasileiro de Informática na Educação (SBIE 2019)

o aluno fique atrás duas ou mais séries indicadas para sua idade [INEP 2019]. Portanto, pode-se notar uma forte relação entre TDI e as variáveis resposta (TA com uma relação inversa). Características dos docentes também mostraram-se influentes, como o índice de professores formados em licenciatura (AFD1) e bacharelado (AFD3) na mesma área que ensinam e a regularidade de ensino que esses professores desempenham nas escolas (IRD). Percebe-se também em ambos os cenários a relação das variáveis resposta com a quantidade de alunos por turma das escolas (ATU). No cenário da aprovação, por fim, é identificada a presença da variável AFD5. Esta variável está relacionada a taxa de professores sem formação superior, indicando que a taxa de professores sem formação apresenta uma relação negativa com a taxa de aprovação das escolas.

\section{Modelos propostos para estimação de indicadores educacionais}

\subsection{Regressão Quantílica Não Paramétrica}

O modelo de RQNP foi executado através do software $\mathrm{R}(R$ Core Team) utilizando o pacote "np" [Hayfield and Racine 2008]. Como função de estimação de parâmetros utilizou-se a função "npcdistbw" proposta por Li et al. (2013) [Li et al. 2013]. Como função de ajuste de modelo de RQNP empregou-se o a função "npqreg" que segundo Koenker [Koenker 2006] o modelo de RQNP é ajustado de acordo com a equação:

$$
\sum_{i=1}^{N} w_{i}(x) \rho_{\tau}\left(y_{i}-\beta_{0}-\beta_{1}\left(x_{i}-x\right)\right)
$$

em que $\rho_{\tau}$ é o quantil escolhido, $y_{i}$ é a variável dependente que se deseja estimar, $x_{i} \mathrm{e}$ $x$ são as variáveis independentes, $\beta_{0}$ e $\beta_{1}$ são os coeficientes de correlação e $w_{i}(x)$ corresponde ao kernel gaussiano, que corresponde à distribuição normal padrão que segundo [Fagundes and Cysneiros 2013], pode ser representado por:

$$
K\left(d\left(x, x_{j}\right)\right)=\frac{1}{(\sqrt{2 \pi})^{1 / p}} \frac{1}{h^{p}} e^{\frac{-d\left(x, c_{i}\right)}{2 h^{2}}}
$$

em que $d\left(x, x_{j}\right)$ é a raiz quadrada da distância euclideana entre $x$ e a localização de interesse $x_{i}$.

\subsection{Otimização por Algoritmos Genéticos}

Os Algoritmos Genéticos (AG) atuaram na estimação do parâmetro Largura de Banda na RQNP com o objetivo de melhorar o desempenho do modelo. A função matemática na qual o parâmetro de suavização está presente é representado pela Equação 2, em que $h$ é o parâmetro à ser otimizado, aqui representado por $h_{D}$, em que $D$ é a dimensão do problema e $h$ é um indivíduo de $D$ dimensões (i.e. uma matriz $1 x D$ ).

A população inicial $P$ é um conjunto de $n$ indivíduos representados por $P=$ $\left(h_{1}, h_{2}, \cdots, h_{D}\right)$. Sabendo que $h_{D}$ é um conjunto de valores entre os limites inferiores e superiores do espaço de busca. Após gerar a população inicial, é necessário avaliar os indivíduos de acordo com a função objetivo, que a RQNP avalia pelo cálculo do Erro Médio Absoluto (i.e Equação 5), após a estimação realizada pela RQNP (Equação 1).

Para seleção de pais foi utilizado o método roleta, que tem como principal característica fornecer maior e menor probabilidade de seleção de indivíduos bons e ruins, 
VIII Congresso Brasileiro de Informática na Educação (CBIE 2019)

Anais do XXX Simpósio Brasileiro de Informática na Educação (SBIE 2019)

respectivamente. A principal vantagem é manter dentro da população indivíduos bons e ruins, pois, os bons têm maior probabilidade de gerar filhos com alta aptidão e os ruins mantém a diversidade na população, desde modo, evitando mínimos locais. A função matemática o método roleta é dado por:

$$
p_{i}=\frac{f_{i}}{\sum_{j=1}^{N} f_{i}}
$$

em que $N$ é o número total de indivíduos na população, $f$ é o resultado retornado pela função objetivo e $p_{i}$ é a probabilidade de seleção do indivíduo.

$\mathrm{Na}$ fase de cruzamento, o método crossover point foi empregado utilizando dois pais para gerar um filho (novo indivíduo da população), escolhendo um ponto aleatório dos cromossomos (pais) e efetuando o cruzamento de informação entre eles para gerar um novo indivíduo. Dado que temos um problema de $D$ dimensões, deverá ser escolhido um ponto $p$ aleatório ente o intervalo $[1, D]$.

Na etapa de mutação, para procurar melhores soluções, uma busca local $b$ foi inserida nos genes dos cromossomos. Em um problema de $D$ dimensões, deverá se escolher um valor inteiro aleatório $y$ entre o intervalo $[1, D]$, este valor selecionado aleatoriamente é a quantidade de genes do cromossomo que sofrerão mutação. Logo após, é necessário selecionar aleatoriamente $y$ genes do cromossomo e aplicar nestes genes uma busca local. A mutação é realizada conforme a Equação 4

$$
h_{D}=h_{D}+b
$$

em que $b$ é um valor aleatório entre o intervalo [-1,1]. Posteriormente, o indivíduo modificado fará parte da próxima geração.

Uma vez realizado os procedimentos de crossover e mutação, os indivíduos da próxima geração $h_{D}$ serão avaliados pela função objetivo elucidada na Equação 5 e um novo ciclo inicia conforme fluxograma da Figura 1.

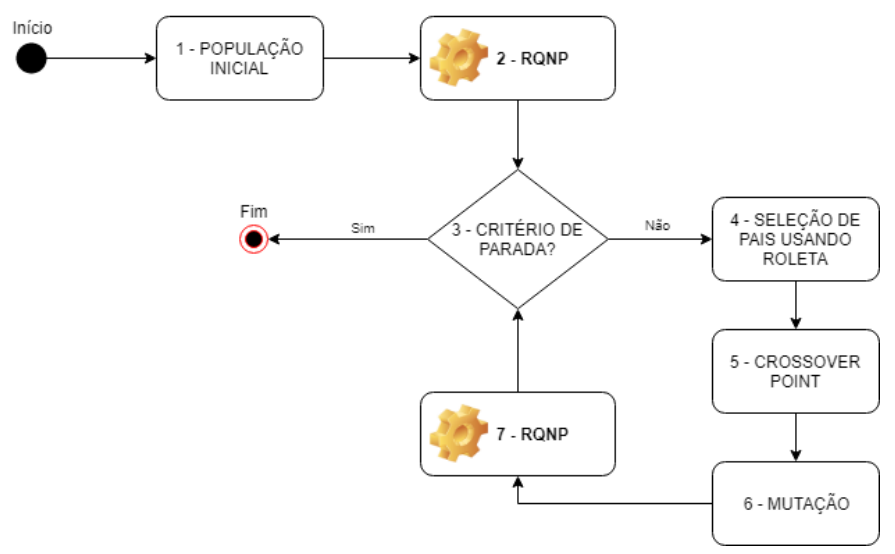

Figura 1. Diagrama Algoritmos Genéticos utilizado para otimizar a RQNP

A Figura 1 mostra um fluxograma do AG presente na literatura. O detalhamento do algoritmo é mostrado de acordo com os passos abaixo. 
VIII Congresso Brasileiro de Informática na Educação (CBIE 2019)

Anais do XXX Simpósio Brasileiro de Informática na Educação (SBIE 2019)

1. Inicializar população aleatoriamente.

2. Calcular fitness da População, utilizando a RQNP apresentada na equação 1.

3. Verifica se o critério de parada foi alcançado. Se sim, finaliza a execução do algoritmo. Se não, executa o próximo passo.

4. Seleção de pais utilizando Roleta, conforme elucidado pela equação 3.

5. Executa crossover point usando os pais selecionados no passo anterior.

6. Executa mutação.

7. Calcular fitness dos indivíduos sobreviventes.

8. Se o critério de parada estiver satisfeito, interrompe a execução do algoritmo. Se não, voltar para o passo 4.

\section{Avaliação Experimental}

Para todos os testes realizados, foram utilizados os seguintes parâmetros: Tamanho da População = 30; Quantidade de iterações $=100$; Chance de ocorrer um crossover $=80 \%$; Chance de ocorrer uma mutação $=5 \%$; Limites inferior e superior do espaço de busca $=$ -1 e 3 (considerados somente na inicialização aleatória da população); Dimensão do problema $=6$; Limites inferior e superior da busca local utilizada nos processos de crossover e mutação $=-1$ e 1, Condições de parada: $(i)$ quando executar o número pré-estabelecido de iterações e (ii) se o algoritmo não evoluir durante $30 \%$ do total de iterações.

A estimação da variável dependente foi realizada utilizando o método de validação cruzada holdout [Kohavi et al. 1995]. Este método separa os dados em dois conjuntos, chamados de treino e teste. Para cada experimento, os dados foram divididos em $75 \%$ como treino e $25 \%$ como conjunto de teste.

A avaliação de desempenho dos modelos baseiam-se na Erro Médio Absoluto $(E M A)$, que é dado pela equação 5:

$$
E M A=\frac{\sum_{i=1}^{n}\left|y_{i}-\hat{y}_{i}\right|}{n}
$$

em que $y_{i}$ é o valor real, $\hat{y}_{i}$ é o valor predito e $n$ é o número de instâncias da base.

O teste estatístico de Wilcoxon para amostras não pareadas com um nível de significância de 5\% foi aplicado, a fim de verificar estatisticamente a superioridade de um modelo sobre outro [Montgomery et al. 2000]. Considerou-se $\mu_{1}$ e $\mu_{2}$ como média EMA dos modelos comparativos. A hipótese nula é $H_{0}: \mu_{1}=\mu_{2}$ e hipótese alternativa é $H_{1}: \mu_{1}<\mu_{2}$. Em que $\mu_{1}$ é a média do resultado da RQNP otimizada por AG e $\mu_{2}$ é a média do resultado da RQNP padrão.

\subsection{Discussões e Resultados}

A Tabela 1 mostra o resultado (quanto menor, melhor) comparativo entre as estimações realizadas pelo modelo RQNP padrão e RQNP otimizado por Algoritmos Genéticos. De acordo com os resultados de média e desvio padrão (entre parêntesis), percebe-se que o modelo otimizado por AG obteve uma média de erro menor com maior desvio padrão. Para avaliar a confiabilidade estatística dos modelos, executamos o teste estatístico de Wilcoxon não pareado com 5\% de significância. O resultado alcançado pelo teste estatístico de Wilcoxon indica que os resultados alcançados pelos modelos são de fato diferentes, $\log$, rejeitamos $H_{0}$. 
VIII Congresso Brasileiro de Informática na Educação (CBIE 2019)

Anais do XXX Simpósio Brasileiro de Informática na Educação (SBIE 2019)

\section{Tabela 1. Resultado comparativo entre a RQNP e os Algoritmos Genéticos, com a média e desvio padrão (entre parêntesis) para o EMA.}

\begin{tabular}{l|l|l}
\hline Conjunto de Dados & RQNP & Algoritmo Genético \\
\hline Aprovação do Ensino Médio & $0.0576(0.0030)$ & $\mathbf{0 . 0 5 2 1}(0.0058)$ \\
\hline Reprovação do Ensino Médio & $0.0834(0.0042)$ & $\mathbf{0 . 0 7 6 4}(0.0129)$ \\
\hline
\end{tabular}

As Figuras 2(a) e 2(b) mostram o resultado comparativo entre a estimação realizada pelo RQNP padrão e a estimação realizada pela RQNP otimizada por AG, nos conjuntos de dados de aprovação e reprovação. De acordo com os gráficos em boxplot apresentados, nota-se que os modelos propostos possuem variações semelhantes, no entanto o modelo otimizado por AG, possui menor mediana amostral.

O modelo otimizado por AG alcança melhor resultado porque enquanto o modelo de RQNP utiliza uma abordagem estatística de estimação baseada em validação cruzada, o AG utiliza uma abordagem de computação inteligente baseada em população, em que para cada geração, 10 indivíduos candidatos são testados, e os melhores resultados são considerados para a próxima geração. Portanto, o modelo com otimizador obteve melhor predição da taxa de reprovação e aprovação escolar no cenário aplicado. O modelo de RQNP padrão, possui menor custo computacional, logo é recomendado em situações que se deseja uma estimação mais rápida. No entanto, se o objetivo é maior precisão de estimação, indica-se a utilização do modelo de RQNP otimizado por AG.

Os modelos propostos neste trabalho podem ser utilizados como sistemas de apoio a decisão, já que indicam em que casos pode haver uma reprovação. Logo, diante da indicação de que pode haver uma alta taxa de reprovação, a escola será capaz de agir preventivamente.

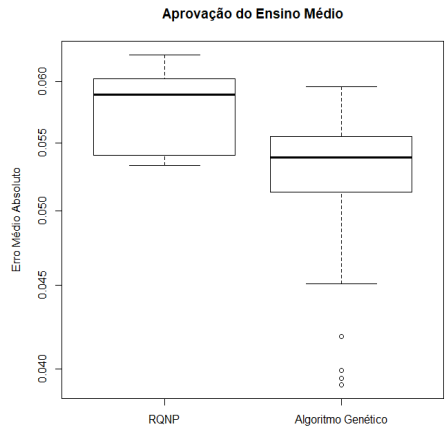

(a) Resultados Aprovação

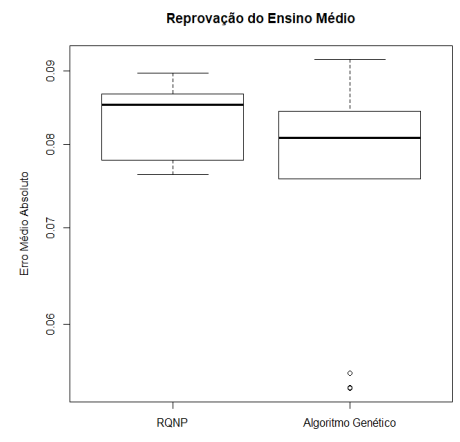

(b) Resultados Reprovação

Figura 2. Boxplot comparativo entre a RQNP e os Algoritmos Genéticos

\section{Conclusões e Trabalhos Futuros}

Este trabalho apresentou duas abordagens para previsão de aprovação e reprovação escolar do ensino médio. O conjunto de dados utilizado neste estudo estão disponibilizadas abertamente pelo INEP, referente ao ano de 2016, para o âmbito do estado de Pernambuco. Este estudo buscou investigar e explicar indicadores educacionais que podem estar relacionadas com a taxa de aprovação e reprovação das escolas. Na seleção de variáveis através da técnica Random Forest, pode ser observado que variáveis como a dispersão 
VIII Congresso Brasileiro de Informática na Educação (CBIE 2019)

Anais do XXX Simpósio Brasileiro de Informática na Educação (SBIE 2019)

idade-série das escolas, nível de formação dos docentes e quantidade de alunos por turma tem maior importância na construção do modelo de estimação (variáveis explicativas).

Outra contribuição deste trabalho são os modelos propostos de Regressão Quantílica Não Paramétrica (RQNP) e RQNP Otimizada por Algoritmos Genéticos (AG) na estimação da aprovação e reprovação escolar. Embora as abordagens apresentem médias parecidas, o teste estatístico de Wilcoxon indica que a abordagem otimizada por Algoritmos Genéticos é capaz de fornecer uma previsão mais precisa, em relação ao modelo sem otimização, uma vez que minimizou o erro de predição.

A Mineração de Dados Educacionais possibilita o conhecimento acerca de variáveis educacionais, resultando em ferramentas que ofereçam melhorias neste cenário. Desta forma, a aplicação de modelos não paramétricos foi satisfatório, oferecendo contribuições através de modelos mais robustos na estimação de índices educacionais. Como trabalhos futuros, pretende-se investigar outros fatores educacionais, assim como aplicar outras formas de otimização de modelos para diminuir o erro da predição.

Através de técnicas de predição (na aplicação da Mineração de Dados Educacionais), sistemas e plataformas podem ser desenvolvidos e adotados no ambiente educacional para fins de identificação de fatores relacionadas as taxas de eficiência escolar, ou quaisquer outros indicadores educacionais. Este trabalho revela o primeiro passo de um estudo que pode ser expandido para auxilio no ensino/aprendizagem, pois identificar fatores influentes na educação pode revelar insights que ajudem a melhorar este cenário, e assim criar programas, intervenções ou investimentos.

\section{Referências}

Baker, R., Isotani, S., and Carvalho, A. (2011). Mineraçao de dados educacionais: Oportunidades para o brasil. Brazilian Journal of Computers in Education, 19(02):03.

Breiman, L. (2001). Random forests machine learning. 45: 5-32. View Article PubMed/NCBI Google Scholar.

Calixto, K., Segundo, C., and de Gusmão, R. P. (2017). Mineração de dados aplicada a educação: um estudo comparativo acerca das características que influenciam a evasão escolar. In Brazilian Symposium on Computers in Education (Simpósio Brasileiro de Informática na Educação-SBIE), volume 28, page 1447.

Colpani, R. (2018). Mineração de dados educacionais: um estudo da evasão no ensino médio com base nos indicadores do censo escolar. Informática na educação: teoria \& prática, 21(3).

Coutinho, L. D. (2015). Utilizando redes neurais artificiais e algoritmos de seleção de variaveis para realizar diagnóstico precoce da doença de alzheimer e do déficit cognitivo leve. Master's thesis, Universidade de Pernambuco.

Darwin, C. (1909). The origin of species. Dent.

do Nascimento, R. L. S., da Cruz Junior, G. G., and de Araújo Fagundes, R. A. (2018a). Mineração de dados educacionais: Um estudo sobre indicadores da educação em bases de dados do inep. RENOTE, 16(1).

do Nascimento, R. L. S., das Neves Junior, R. B., de Almeida Neto, M. A., and de Araújo Fagundes, R. A. (2018b). Educational data mining: An application of regres- 
VIII Congresso Brasileiro de Informática na Educação (CBIE 2019)

Anais do XXX Simpósio Brasileiro de Informática na Educação (SBIE 2019)

sors in predicting school dropout. In International Conference on Machine Learning and Data Mining in Pattern Recognition, pages 246-257. Springer.

Fagundes, R. A. d. A. and Cysneiros, F. J. d. A. (2013). Métodos de regressão robusta e kernel para dados intervalares. Universidade Federal de Pernambuco.

Hayfield, T. and Racine, J. S. (2008). Nonparametric econometrics: The np package. Journal of Statistical Software, 27(5).

Holland, J. H. (1992). Adaptation in natural and artificial systems: an introductory analysis with applications to biology, control, and artificial intelligence. MIT press.

INEP (2019). Instituto nacional de estudos e pesquisas educacionais anísio teixeira. url: http://portalinep.gov.br/. Accesso em 14 de Janeiro de 2019.

Koenker, R. (2006). Quantile regresssion. Encyclopedia of environmetrics.

Koenker, R. and Bassett Jr, G. (1978). Regression quantiles. Econometrica: journal of the Econometric Society, pages 33-50.

Kohavi, R. et al. (1995). A study of cross-validation and bootstrap for accuracy estimation and model selection. In Ijcai, volume 14, pages 1137-1145. Montreal, Canada.

Laisa, J. and Nunes, I. (2015). Mineração de dados educacionais como apoio para a classificação de alunos do ensino médio. In Brazilian Symposium on Computers in Education (Simpósio Brasileiro de Informática na Educação-SBIE), volume 26, page 1112.

Li, Q., Lin, J., and Racine, J. S. (2013). Optimal bandwidth selection for nonparametric conditional distribution and quantile functions. Journal of Business \& Economic Statistics, 31(1):57-65.

Montgomery, D. C., Peck, E. A., and Vining, G. G. (2012). Introduction to linear regression analysis, volume 821.

Montgomery, D. C., Runger, G. C., and Calado, V. (2000). Estatística Aplicada E Probabilidade Para Engenheiros. Grupo Gen-LTC.

Romero, C. and Ventura, S. (2010). Educational data mining: a review of the state of the art. IEEE Transactions on Systems, Man, and Cybernetics, Part C (Applications and Reviews), 40(6):601-618. 